\title{
Diversité botanique dans le sud du parc national de Taii, Côte d'Ivoire
}

\author{
C. Y. Adou Yao* et E. K. N'Guessan \\ UFR Biosciences - Laboratoire de Botanique, Université de Cocody - Abidjan, 22 BP 582, \\ ABIDJAN 22, Côte d'Ivoire.
}

(Reçu le 11 Avril 2005, accepté le 22 Juillet 2005)

* Correspondance et tirés à part, e-mail : adouyaocy@yahoo.fr

\section{Résumé}

Le Parc National de Taï (PNT) constitue l'un des derniers vestiges de forêt ombrophile " primaire "I d'Afrique de l'Ouest. Si la partie septentrionale est relativement bien connue, la partie méridionale l'est moins. Pour pallier ce déficit, une étude de la diversité botanique du Sud-PNT a été conduite. Ce recensement s'est réalisé par deux méthodes complémentaires : le relevé itinérant et le relevé de surface. L'inventaire a permis de cataloguer 908 espèces de plantes de toutes les tailles qui viennent compléter la liste des espèces de Taii à 1231 espèces. Ces différentes espèces se répartissent en 550 genres et 116 familles: les mieux représentées sont les Rubiaceae, les Euphorbiaceae et les Caesalpiniaceae. Parmi toutes les espèces inventoriées, 175 sont endémiques, 11 sont endémiques ivoiriennes et 61 sont espèces "sassandriennes".

L'étude a montré aussi que pour les arbres de diamètre $\geq 10 \mathrm{~cm}$, les espèces Hymenostegia afzelii, Strombosia pustulata, Diospyros sanza-minika, Funtumia africana et Calpocalyx brevibracteatus et des représentants de Caesalpiniaceae, Euphorbiaceae et Ebenaceae sont celles qui ont les plus fortes valeurs d'indice de valeur d'importance des espèces (IVI) et d'indice de valeur d'importance des familles (VIF). Le nombre élevé d'espèces endémiques, "sassandriennes", rares et menacées que contient cette flore, représente sa caractéristique particulière majeure par rapport aux autres forêts ivoiriennes et confirme que le PNT appartient au centre de grande diversité d'Afrique de l'Ouest (Guinean Forests of West Africa Hotspot).

Mots-clés : Diversité botanique, Endémiques, Importance relative des familles, Parc National de Taï 


\section{ABSTRACT \\ Botanical diversity of the South of Taï National Park, Côte d'Ivoire}

The Tai National Park (TNP) is one of the last vestiges of " primary " forest remained in West Africa. The northern part is almost well known but the southern one is not. To mitigate this lack, we conducted a study in the South of the TNP. To achieve the inventory, two methods were used: "walk set up" and the "plot set up". Nine hundred height plants species were recorded. They belonged to 550 genera and 116 families. The richest families were Rubiaceae, Euphorbiaceae and Caesalpiniaceae. Among all the species, 175 were endemic of West African forests, 11 endemic to Ivorian forests and 61 "sassandrian". The study showed that for tree species with $\mathrm{dbh} \geq 10 \mathrm{~cm}$ Hymenostegia afzelii, Strombosia pustulata, Diospyros sanza-minika, Funtumia africana, Calpocalyx brevibracteatus and representatives of Caesalpiniaceae, Euphorbiaceae and Ebenaceae families were the richest and had the highest values of IVI and FIV. Endemic, "sassandrian", rare and threatened species constitute the major characteristic of the TNP and confirm that it includes in the Guinean Forests of West Africa Hotspot.

Keywords : Botanical diversity, Endemic, Family importance value, Taï National Park.

\section{Introduction}

En qualifiant de "primaire" le massif forestier occupant le Sud-0uest de la Côte d'Ivoire, de nombreux auteurs [1,2] ont largement contribué à attirer sur lui, l'attention de tous les aménageurs et protecteurs de la nature. Il est décrit comme l'une des dernières forêts denses encore intacte en Côte d'Ivoire, l'un des derniers vestiges de forêt "primaire" s'étant maintenu après les grandes périodes de glaciation des aires quaternaires et tertiaires. C'est pourquoi le PNT a été intégré par l'UNESCO dans le Réseau des "Réserves de la Biosphère" en 1978. II l'a inscrit en 1981, sur la liste des sites du "Patrimoine Naturel Mondial de l'Humanité" [3].

La forêt du PNT appartient, comme la plupart des forêts du Sud-Ouest ivoirien, à la région phytogéographique Guinéo-Congolaise. Sa richesse floristique est estimée à 1300 espèces dont 150 espèces sont endémiques au massif $[4,5]$. Cette richesse spécifique est tout aussi impressionnante au niveau des animaux que des plantes. Ces richesses naturelles, mais aussi les pressions qui pèsent sur elles, ont amené Conservation International en 1999 à inscrire le Parc dans la catégorie des "hotsponl de biodiversité. Le Parc a ainsi été zone prioritaire de conservation des animaux et des plantes. 
Hormis Guillaumet [6] qui a montré l'existence de plusieurs espèces endémiques et rares dans l'ensemble du Sud-Ouest ivoirien, les seules études traitant de la végétation de la partie Sud du Parc sont celles de Huttel [7] et de Van Rompaey [8]. La plupart des travaux concerne soit la faune $[1,9,10]$ soit les relations faune-flore $[2,11]$. De plus la plupart de ces études sont des inventaires qui ne portent que sur les ligneux de plus de $10 \mathrm{~cm}$ de diamètre [12]. Cette insuffisance de travaux est encore plus marquée dans la partie Sud où très peu de récoltes botaniques et de relevés ont été faits, depuis son intégration au Parc, il y a 28 ans. Et pourtant, le sud du Parc est très proche du massif de Grabo, un autre 'hotspot'de biodiversité.

La présente étude qui est l'une des premières à se dérouler dans la partie Sud du PNT se fixe pour objectif principal d'inventorier et d'évaluer la diversité botanique du Sud du PNT.

\section{Méthodologie}

Pour la collecte des données, notre site d'étude a été divisée en trois secteurs (Figure I) : le Sud-Ouest (Guiroutou) du Parc, le Sud-Sud (Dogbo) et le Sud-Est (Djapadii). La collecte des données s'est effectuée d'avril 1998 à janvier 1999. Ceci nous a permis d'explorer la zone d'étude à différentes périodes de l'année, afin de pouvoir inventorier le maximum d'espèces.

\section{2-1. Milieu d'étude}

Le Parc National de Taï, au Sud-Ouest de la Côte d'Ivoire, est compris entre les latitudes $5^{\circ} 10^{\prime}$ et $6^{\circ} 50^{\prime}$ Nord et les longitudes $6^{\circ} 50^{\prime}$ et $7^{\circ} 50^{\prime}$ Ouest (Figure 1). Sa limite ouest est située à $20 \mathrm{~km}$ à l'Est de la ville de Taï et sa limite sud à environ $60 \mathrm{~km}$ au Nord de la ville de Grabo. II s'étend entre les isohyètes $1700 \mathrm{~mm}$ au Nord et $2200 \mathrm{~mm}$ au Sud, sur 457.261 ha (environ $100 \mathrm{~km}$ de long et $50 \mathrm{~km}$ de large) [13]. La zone Sud, comprise entre la rivière Hana et la limite sud du parc, couvre environ 100.000 ha, soit environ le quart de la surface du Parc.

Le Parc National de Taï est soumis à un climat équatorial de transition, typique de la région située au-dessous du $8^{e}$ parallèle [14]. La pluviosité moyenne annuelle dans la région de Taii de 1980 à 1997 est de 1976,14 mm. Elle varie de $1700 \mathrm{~mm}$ au Nord à 2200 mm au Sud du Parc [15].

Le relief, dans le parc est constitué d'un ensemble de collines, assez uniforme et sillonné par de nombreux cours d'eau très ramifiés [16]. S'y ajoutent quelques plateaux, qui atteignent à peine 150 à $200 \mathrm{~m}$ d'altitude. Les bas-fonds sont plats, larges de 100 à $150 \mathrm{~m}$, marécageux et de pente longitudinale faible [17]. La monotonie générale est à 
peine interrompue par un relief particulier avec notamment le Mont Niénokoué qui culmine à $396 \mathrm{~m}$.

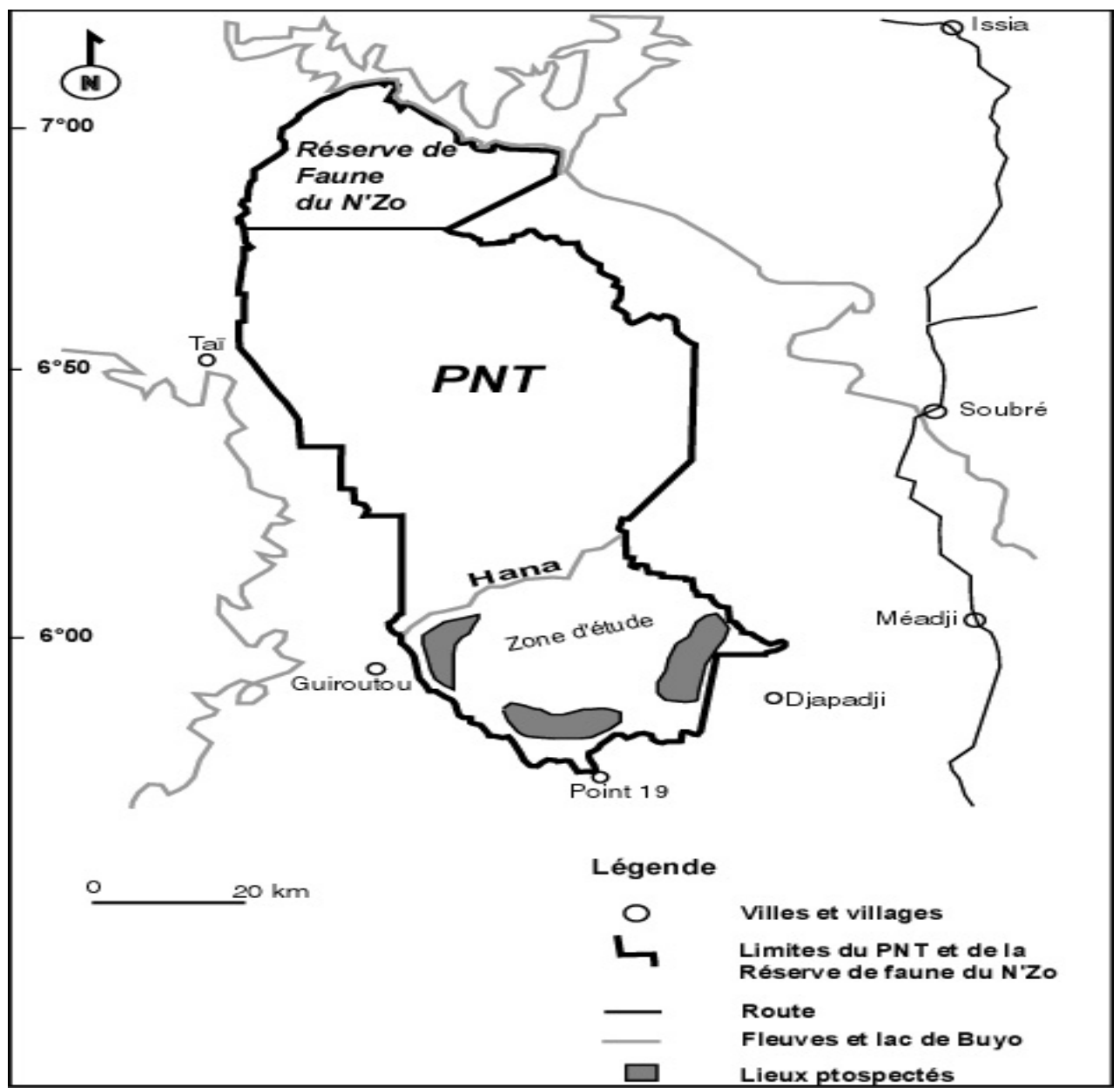

Figure 1 : Localisation de la zone d'étude dans le sud du Parc National de Taï.

Dans le Sud du Parc National de Taï, la texture des sols est sableuse fine et argileuse ou argilo-sableuse. Généralement fertiles, ces sols sont propices aux cultures vivrières (manioc, riz pluvial, bananier, plantain) et cultures de rentes (cacaoyers, caféiers, palmiers à huile, hévéas).

La forêt du PNT est dense sempervirente de basse altitude. Son originalité est, selon Aké Assi [18], marquée par un cortège d'espèces endémiques à la région de la Haute Guinée et à la Côte d'Ivoire : Androsiphonia adenostegia, Vepris tabovensis, 
Triphyophyllum peltatum, Campylospermum amplectens et Cola caricifolia. Ce même auteur faisait remarquer que la richesse du PNT ne pouvait toujours pas être totalement chiffrée, d'où l'intérêt du présent travail.

\section{2-2. Inventaire}

Deux méthodes de collecte ont été utilisées : le relevé de surface et le relevé itinérant.

Pour le premier, nous avons choisi la méthode classique de Hall et Swaine [19] : elle permet de recenser un maximum d'espèces; elle a déjà été utilisée notamment au Ghana par Hawthorne et Abu-Juam [20] et Hawthorne [21] et au Cameroun par Cheek et Cable [22].

Elle a consisté en la pose de placettes carrées de $25 \times 25 \mathrm{~m}$ dans les différentes formations végétales. Au total, 39 placettes ont été positionnées de façon aléatoire : 13 à Guiroutou, 15 à Dogbo et 11 à Djapadji. Pour des difficultés d'accès à la zone d'étude, il n'a pas été possible d'installer le même nombre de placettes dans les trois secteurs. La topographie du terrain (sommet, versant, bas-fond et plaine) et la physionomie de la végétation (nombre de strates, densité relative des plantes dans les différentes strates) ont été les principaux descripteurs qui ont guidé le choix des placettes. Dans chaque placette, la liste de toutes les espèces (arbres, arbustes, arbrisseaux, lianes et herbes) a été dressée.

Les inventaires itinérants ou relevés de parcours ont été essentiellement réalisés, secteur par secteur, le long des layons de surveillance aménagés par les forestiers du Parc. Dans le but de compléter la liste floristique générale du Sud du PNT, on s'est efforcé de noter toutes les plantes qui n'avaient pas été rencontrées dans les placettes.

\section{2-3. Analyse des données}

Pour chacune des 39 placettes, et pour chacun des trois secteurs (Djapadii, Dogbo, Guiroutou), le nombre total d'espèces a été compté. Par comparaison de nos listes avec celles établies par Aké Assi [23,24], nous avons dressé la liste des espèces endémiques du Sud du PNT. Nous nous sommes aussi servis des listes de Châtelain et al. [12] qui ont trouvé 80 espèces endémiques dans l'ensemble du Parc. Nous nous sommes enfin référés aux travaux encore plus récents de Jongkind [25].

Pour les individus d'arbre et de liane, leurs dbh (diamètre à hauteur de la poitrine, soit $1,30 \mathrm{~m}$ au-dessus du sol) atteignant au moins $10 \mathrm{~cm}$ ont été notés. Le nombre d'espèces de ces individus a été compté. Les données sur les arbres à $\mathrm{dbh} \geq 10 \mathrm{~cm}$, ont permis de calculer deux indices pour évaluer la diversité botanique : la Valeur d'Importance des espèces (IVI) et la valeur d'importance des familles (VIF). 
L'Indice de Valeur d'Importance des espèces (IVI) a été mis au point par Curtis et MacIntosh [26]. II caractérise l'importance, au sein d'une végétation, d'une espèce par rapport à l'ensemble des autres espèces. II a été fréquemment utilisé pour évaluer la prépondérance spécifique en forêts tropicales $[27,28]$. Son équivalent pour les familles a été proposé par Cottam et Curtis [29]. II permet de calculer la valeur d'importance des familles (VIF) et a été notamment utilisé par certains Auteurs [27,30].

$$
\text { IVI = FREQesp + DENSesp + DOMesp }
$$

La fréquence relative d'une espèce (FREQesp) est le rapport de sa fréquence spécifique par le total des fréquences spécifiques de toutes les espèces multiplié par cent. La fréquence spécifique d'une espèce est le nombre de placettes dans lesquelles cette espèce est présente.

La densité relative d'une espèce (DENSesp) est le rapport de sa densité absolue (c'està-dire le nombre d'individus par unité de surface) au total des densités absolues de toutes les espèces.

La dominance relative d'une espèce (DOMesp) est le quotient de son aire basale (c'est-à-dire la surface de la section du tronc correspondant au dbh) avec l'aire basale totale de toutes les espèces.

$$
\text { VIF = FREQfam + DENSfam + DOMfam }
$$

La fréquence relative d'une famille (FREQfam) est le rapport de la fréquence des espèces de cette famille au total des fréquences spécifiques de toutes les familles.

La densité relative d'une famille (DENSfam) est le rapport de la densité absolue des espèces de cette famille (c'est-à-dire le nombre d'individus par unité de surface) au total des densités absolues de toutes les espèces.

La dominance relative d'une famille (DOMfam) est le quotient des aires basales des espèces appartenant à cette famille (c'est-à-dire la surface de la section du tronc correspondant au dbh) avec l'aire basale totale de toutes les espèces.

\section{Résultats}

\section{3-1. Composition floristique}

A l'issue de cette étude, 908 espèces ont été inventoriées : 787 (86,7\%) dans les placettes et 121 (13,3\%) lors des relevés itinérants. Ces 908 espèces se répartissent en 116 familles. Les plus riches sont celles des Rubiaceae et Euphorbiaceae dans chaque secteur (Tableau 1). Secteur par secteur, le nombre de familles (88) à Djapadji est plus 
élevé qu'à Guiroutou (85) et Dogbo (80). Le secteur de Guiroutou est le plus riche en espèces, suivi par Djapadji et Dogbo.

Tableau 1 : Répartition des espèces recensées dans le Sud du PNT par secteur, par famille, par type morphologique et par placettes et relevés itinérants.

\begin{tabular}{|c|c|c|c|c|}
\hline & & Dogbo & Guiroutou & Djapadji \\
\hline $\begin{array}{l}\text { Nombre total } \\
\text { d'espèces }\end{array}$ & Total par site & 547 & 660 & 609 \\
\hline \multirow[t]{5}{*}{908} & Relevés itinérants & 93 & 105 & 153 \\
\hline & Placettes & 459 & 568 & 461 \\
\hline & Maximum/placette & 198 & 151 & 169 \\
\hline & Minimum/placette & 59 & 67 & 88 \\
\hline & Moyenne/placette & $134,1 \pm 35,3$ & $118,8 \pm 26,5$ & $135,0 \pm 21,3$ \\
\hline $\begin{array}{l}\text { Nombre total de } \\
\text { familles } 116\end{array}$ & Total par site & 80 & 85 & 88 \\
\hline Types & Arbres et arbustes & 256 & 280 & 290 \\
\hline \multirow[t]{2}{*}{ morphologiques } & Lianes & 166 & 129 & 146 \\
\hline & Fougères & 17 & 24 & 11 \\
\hline
\end{tabular}

Lorsque nous considérons les espèces récoltées uniquement dans les placettes, Guiroutou est également le plus riche des trois secteurs. Dogbo et Djapadji sont sensiblement riches l'un comme l'autre. La moyenne d'espèces par placette est plus élevée à Djapadji $(135,0 \pm 21,3)$ et Dogbo $(134,1 \pm 35,3)$ qu'à Guiroutou $(118,8 \pm 26,5)$. Le maximum d'espèces par placette a été rencontré à Dogbo mais c'est le secteur où l'on a rencontré la placette la moins riche en espèce de cette étude.

L'application simultanée de deux techniques de relevés nous a permis d'obtenir des inventaires plus complets (Tableau 1). Ce sont les relevés par placettes qui donnent les meilleurs résultats. Mais dans le cas du secteur de Djapadji, le relevé itinérant a permis de révéler $1 / 4$ des espèces en plus : $74,4 \%$ dans les 11 placettes et $25,6 \%$ en itinérant. Dans les deux autres secteurs, les espèces inventoriées par relevés itinérants sont moins nombreuses: pour Dogbo, 80,8\% dans les 15 placettes et $19,2 \%$ en relevé itinérant; pour Guiroutou, $85,9 \%$ dans les 13 placettes et $14,1 \%$ en relevé itinérant. 
Cela est sans doute dô au fait que la végétation de Djapadji, plus ouverte se prête mieux à l'inventaire itinérant.

Parmi les espèces, 501 (55\%) sont des arbres et arbustes, 221 (24,3\%) des lianes et $38(4,2 \%)$ des fougères ; le reste est constitué d'herbes, chaméphytes, géophytes.

La courbe aire-espèces (Figure 2) pour l'ensemble du sud du PNT est toujours croissante, même lorsque les 39 placettes ont été prises en compte. On constate cependant, que la courbe a tendance à former un plateau, ce qui signifierait que l'on se rapproche de cette aire minimale.

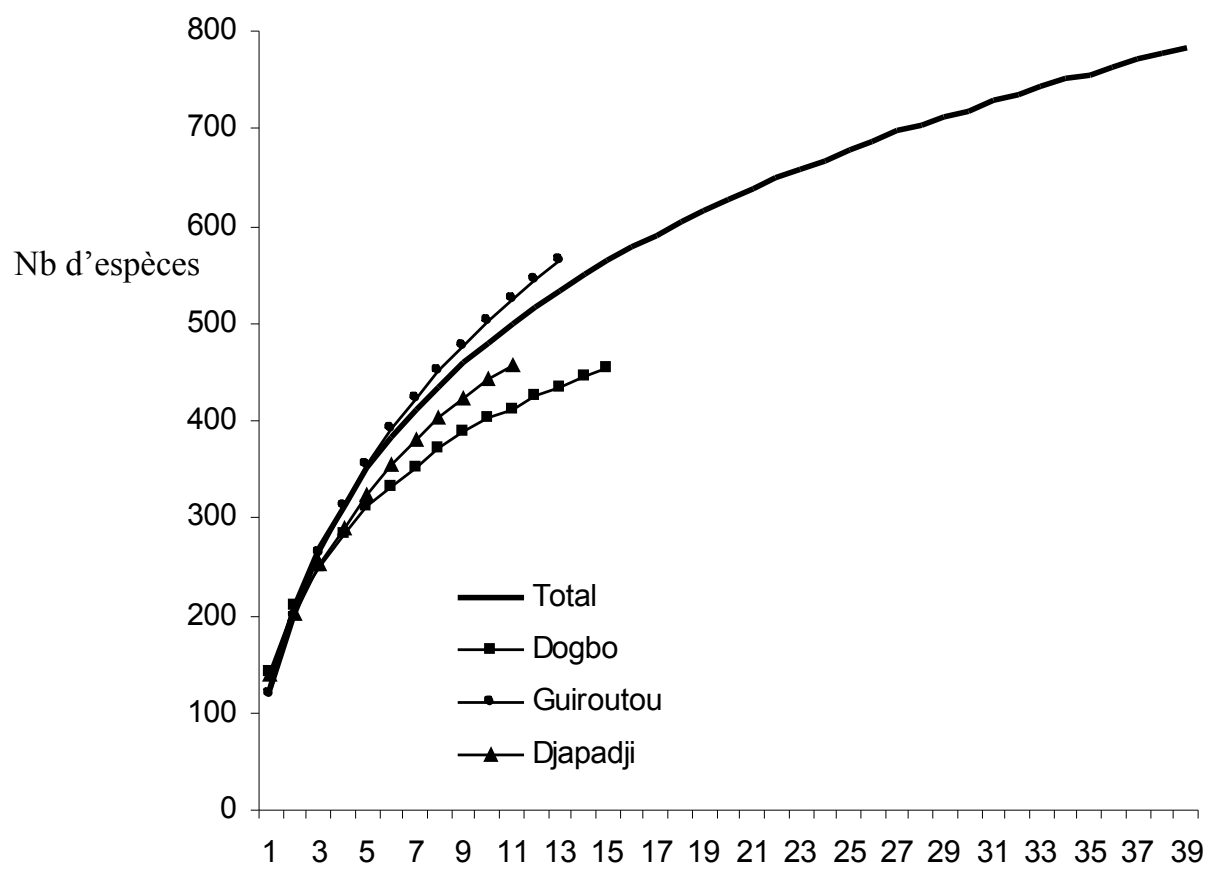

Numéros de placette

Figure 2 : Courbes aire-espèces des plantes inventoriées dans chaque secteur d'étude et dans l'ensemble du Sud-PNT.

En ce qui concerne les familles, les mieux représentées sont celles des Rubiaceae, des Euphorbiaceae et des Caesalpiniaceae à Dogbo, des Rubiaceae, des Euphorbiaceae, des Annonaceae, des Apocynaceae et des Caesalpiniaceae à Guiroutou et des Rubiaceae, des Euphorbiaceae, Caesalpiniaceae et Annonaceae à Djapadji. Comme la plupart des forêts 
tropicales africaines, la forêt du Parc est donc du type "Rubiaceae - Euphorbiaceae Caesalpiniaceae".

\section{3-2. Les espèces rares et endémiques dans le Sud du PNT}

Les 908 espèces de notre liste ne sont pas également représentées à l'intérieur du Parc. Deux cent trente cinq n'apparaissent que dans une seule placette et 134 dans seulement 2 placettes : on peut donc estimer à $40,6 \%$, la proportion d'espèces peu fréquentes. A l'opposé, les plus fréquentes, qui apparaissent dans au moins 30 placettes à la fois, sont au nombre de 15 (soit $1,7 \%$ ). La plus fréquente de toutes les espèces est un ligneux, Strombosia pustulata, présent dans 37 placettes sur 39 : c'est une essence ordinaire, fréquente dans la plupart des couverts forestiers ivoiriens.

Lorsqu'on compare les listes des 3 secteurs, on s'aperçoit qu'ils ont en commun 334 espèces : cet ensemble peut être considéré comme le fond floristique du Sud du Parc. Le secteur de Guiroutou contient en propre 174 espèces, alors que Djapadji, n'en contient que 88 et Dogbo 66 espèces.

La flore de notre site d'étude contient 175 espèces endémiques qui se répartissent, pour reprendre la classification de Aké Assi [31], en 11 ivoiriennes (Guinéo-Congolaises Ivoiriennes, GCl) : Albertisia cordifolia, Salacia columna var. akeassi et 164 espèces endémiques ouest-africaines (Guinéo-Congolaises Ouest-Africaines, GCW) : Dracaena cristula, Mapania baldwinii, Oncoba brevipes. Parmi ces 175 endémiques, 61 sont sassandriennes (Tableau 2), c'est-à-dire qu'on ne les trouve que dans cette partie de la Côte d'Ivoire. Le secteur de Guiroutou contient le plus grand nombre d'espèces endémiques et sassandriennes (Tableau 2).

Le Sud-PNT contient 247 espèces endémiques de la région phytogéographique de Haute Guinée: c'est encore Guiroutou qui est le secteur le plus riche et Dogbo le plus pauvre.

Dans la liste générale, on retrouve 11 espèces qui figurent dans la liste des raretés (c'est-à-dire des espèces dont les usages locaux entraîne une réduction drastique de leur nombre dans la flore ivoirienne) botaniques pour l'ensemble de la Côte d'Ivoire, que Aké Assi $[31,32]$ a dressée : en particulier, les lianes Albertisia cordifolia et $A$. mangenotii, connues seulement dans le Parc du Banco et la forêt d'Adiopodoumé et aussi l'arbre Senna aubrevillei (espèce d'ailleurs encore mal connue sur le plan taxonomique) et la sous-espèce akeassii de Salacia columna. 
Tableau 2 : Répartition des espèces du sud du PNT selon leur endémisme (GCl, GCW, Sassandriennes, stars du Ghana et distribution selon Jongkind [25].

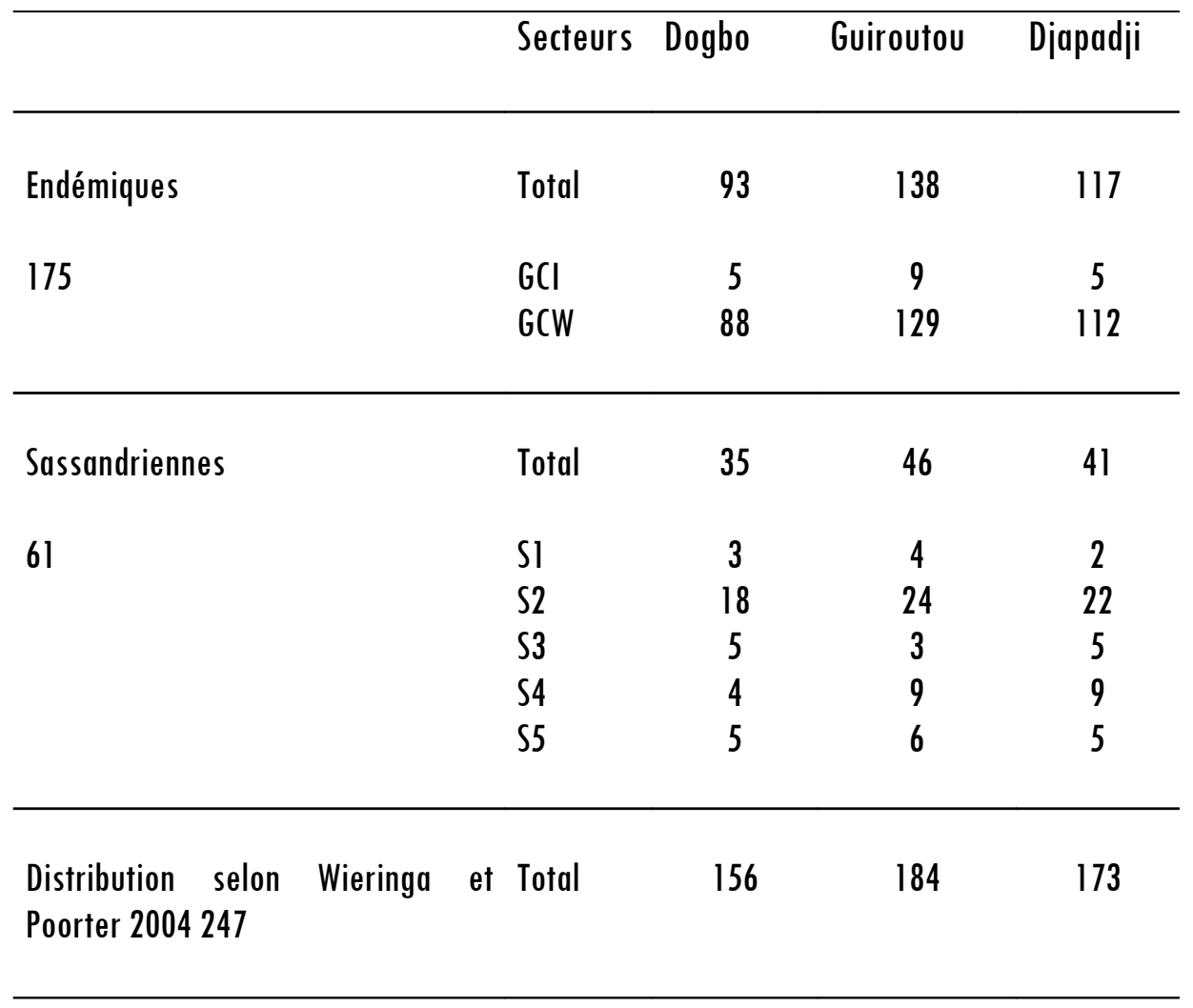

GCI: Guinéo-Congolaise Ivoiriennes, GCW Guinéo-Congolaise Ouest-africaine.

$S 1, \ldots, S 5$ : Espèces sassandriennes des groupes $1,2, \ldots, 5$.

IId : espèces endémiques de la région phytogéographique de la Haute Guinée

\section{3-3. Importance relative des espèces et familles}

Lorsqu'on considère uniquement les grandes plantes (arbres, arbustes et lianes de diamètre $\geq 10 \mathrm{~cm}$ ), l'inventaire a permis de recenser seulement 155 espèces. Les 5 espèces dont les valeurs d'importance (IVI) sont les plus élevées (de 14 à 30) sont Hymenostegia afzelii, Strombosia pustulata, Diospyros sanza-minika, Funtumia africana et Calpocalyx brevibracteatus (Tableau 3). Nous les qualifions à la suite de Mori et al. [27] et Kouamé [28] d'espèces prépondérantes générales. Hymenostegia afzelii possède I'IVI le plus élevé : elle ne se rencontre pourtant que dans 10 placettes sur 39 mais elle y est représentée par un très grand nombre d'individus (112 individus sur un total de 
1165 , toutes espèces confondues) : il s'agit donc typiquement d'une espèce grégaire. Les autres essences à IVI forts se rencontrent dans un grand nombre de placettes.

Tableau 3 : Les espèces prépondérantes des différents sites et de tout le Sud PNT selon leur Indice de Valeur d'Importance d'espèces (IVI).

\begin{tabular}{|c|c|c|c|c|c|}
\hline & Espèces & $\begin{array}{l}\text { Fréquence } \\
\text { relative }\end{array}$ & $\begin{array}{l}\text { Densité } \\
\text { relative }\end{array}$ & $\begin{array}{l}\text { Dominance } \\
\text { relative }\end{array}$ & IVI \\
\hline \multirow[t]{11}{*}{ Dogbo } & Hymenostegia afzelii & 3,70 & 16,43 & 6,58 & 26,71 \\
\hline & Strombosia pustulata & 4,32 & 8,22 & 6,70 & 19,23 \\
\hline & Xylopia quintasii & 3,70 & 8,22 & 3,92 & 15,84 \\
\hline & $\begin{array}{l}\text { Scottelia klaineana var. } \\
\text { mimfiensis }\end{array}$ & 4,32 & 4,25 & 3,42 & 11,99 \\
\hline & Scytopetalum tieghemii & 3,09 & 4,25 & 4,08 & 11,42 \\
\hline & Oldfieldia africana & 3,09 & 3,12 & 2,98 & 9,19 \\
\hline & Coula edulis & 3,09 & 2,83 & 2,19 & 8,11 \\
\hline & Diospyros sanza-minika & 1,85 & 2,55 & 2,53 & 6,94 \\
\hline & $\begin{array}{l}\text { Scottelia klaineana var. } \\
\text { klaineana }\end{array}$ & 2,47 & 1,98 & 2,09 & 6,55 \\
\hline & Calpocalyx brevibracteatus & 2,47 & 2,55 & 1,23 & 6,25 \\
\hline & Autres & 67,90 & 45,60 & 64,26 & 177,78 \\
\hline \multirow[t]{11}{*}{ Guiroutou } & Sacoglottis gabonensis & 2,81 & 1,11 & 16,61 & 20,53 \\
\hline & Funtumia africana & 2,25 & 9,98 & 5,88 & 18,11 \\
\hline & Hymenostegia afzelii & 2,25 & 12,20 & 3,04 & 17,48 \\
\hline & Calpocalyx brevibracteatus & 3,37 & 6,21 & 3,46 & 13,04 \\
\hline & Strombosia pustulata & 3,37 & 5,10 & 2,44 & 10,91 \\
\hline & Diospyros sanza-minika & 2,25 & 5,10 & 2,87 & 10,21 \\
\hline & Ricinodendron heudelotii & 1,12 & 0,44 & 5,89 & 7,46 \\
\hline & Octoknema borealis & 3,37 & 2,44 & 1,40 & 7,21 \\
\hline & Protomegabaria stapfiana & 1,69 & 2,44 & 3,04 & 7,17 \\
\hline & Uapaca esculenta & 1,69 & 0,89 & 4,11 & 6,68 \\
\hline & Autres & 75,84 & 54,10 & 51,26 & 181,20 \\
\hline \multirow[t]{11}{*}{ Djapadii } & Diospyros sanza-minika & 5,46 & 10,80 & 4,89 & 21,16 \\
\hline & Strombosia pustulata & 4,92 & 4,71 & 3,80 & 13,43 \\
\hline & Keayodendron bridelioides & 3,28 & 3,32 & 4,52 & 11,12 \\
\hline & Calpocalyx brevibracteatus & 2,73 & 4,99 & 2,31 & 10,03 \\
\hline & Diospyros cananiculata & 3,28 & 4,43 & 1,28 & 8,99 \\
\hline & Diospyros soubreana & 2,73 & 5,26 & 0,89 & 8,89 \\
\hline & Xylopia quintasii & 2,73 & 3,32 & 2,62 & 8,67 \\
\hline & Calpocalyx aubrevillei & 1,64 & 1,94 & 4,44 & 8,02 \\
\hline & Tarrietia utilis & 1,64 & 1,66 & 4,47 & 7,78 \\
\hline & Funtumia africana & 1,09 & 4,16 & 2,52 & 7,76 \\
\hline & Autres & 70,50 & 65,65 & 68,26 & 194,17 \\
\hline
\end{tabular}




\begin{tabular}{|c|c|c|c|c|c|}
\hline & Espèces & $\begin{array}{l}\text { Fréquence } \\
\text { relative }\end{array}$ & $\begin{array}{l}\text { Densité } \\
\text { relative }\end{array}$ & $\begin{array}{l}\text { Dominance } \\
\text { relative }\end{array}$ & IVI \\
\hline \multirow[t]{11}{*}{$\overline{\text { Sud du PNT }}$} & Hymenostegia afzelii & 1,95 & 9,71 & 18,64 & 30,29 \\
\hline & Strombosia pustulata & 4,28 & 5,98 & 13,17 & 23,43 \\
\hline & Diospyros sanza-minika & 3,31 & 6,15 & 12,00 & 21,46 \\
\hline & Funtumia africana & 1,75 & 5,46 & 10,81 & 18,02 \\
\hline & Calpocalyx brevibracteatus & 2,92 & 4,77 & 6,63 & 14,31 \\
\hline & Xylopia quintasii & 2,72 & 3,90 & 5,15 & 11,77 \\
\hline & Scytopetalum tieghemii & 2,72 & 2,60 & 2,73 & 8,06 \\
\hline & $\begin{array}{l}\text { Scottelia klaineana var. } \\
\text { klaineana }\end{array}$ & 2,33 & 2,17 & 1,92 & 6,42 \\
\hline & $\begin{array}{l}\text { Scottelia klaineana var. } \\
\text { mimfiensis }\end{array}$ & 2,53 & 2,08 & 1,78 & 6,39 \\
\hline & Protomegabaria stapfiana & 1,36 & 1,91 & 3,11 & 6,38 \\
\hline & Autres & 74,12 & 55,29 & 24,06 & 153,49 \\
\hline
\end{tabular}

Lorsque l'on compare les différents secteurs étudiés, on constate que parmi les $22 \%$ d'espèces communes, 3 espèces seulement appartiennent à la liste des essences prépondérantes générales : il s'agit de Diospyros sanza-minika, Strombosia pustulata et Calpocalyx brevibracteatus. Chaque secteur a ses propres espèces prépondérantes (Tableau 3). A Dogbo par exemple, les 2 espèces prépondérantes sont Hymenostegia afzelii et Strombosia pustulata ; à Guiroutou, ce sont Sacoglottis gabonensis et Funtumia africana et à Djapadii, ce sont : Diospyros sanza-minika et Strombosia pustulata. La forte dispersion, caractère commun de toutes les forêts tropicales, est confirmée par nos résultats.

En ce qui concerne le VIF, les 2 familles prépondérantes du Sud-PNT sont les Caesalpiniaceae et les Euphorbiaceae (Tableav 4).

Tableau 4 : Les familles prépondérantes des différents sites et de tout le Sud PNT selon leur Indice de Valeur d'Importance des familles (VIF).

\begin{tabular}{llcccc}
\hline Familles & $\begin{array}{c}\text { Diversité } \\
\text { relative }\end{array}$ & $\begin{array}{c}\text { Densité } \\
\text { relative }\end{array}$ & $\begin{array}{c}\text { Dominance } \\
\text { relative }\end{array}$ & VIF \\
\hline Dogbo & Caesalpiniaceae & 8.9 & 20.4 & 21.77 & 51.02 \\
& Annonaceae & 6.3 & 11.0 & 7.85 & 25.23 \\
& Euphorbiaceae & 11.4 & 7.1 & 5.46 & 23.94 \\
& Olacaceae & 3.8 & 11.6 & 7.95 & 23.36
\end{tabular}




\begin{tabular}{|c|c|c|c|c|c|}
\hline & Familles & $\begin{array}{l}\text { Diversité } \\
\text { relative }\end{array}$ & $\begin{array}{l}\text { Densité } \\
\text { relative }\end{array}$ & $\begin{array}{c}\text { Dominance } \\
\text { relative }\end{array}$ & VIF \\
\hline & Ebenaceae & 5.1 & 7.4 & 6.09 & 18.52 \\
\hline & Mimosaceae & 7.6 & 5.1 & 5.55 & 18.25 \\
\hline & Flacourtiaceae & 3.8 & 7.4 & 6.01 & 17.18 \\
\hline & Sterculiaceae & 6.3 & 3.4 & 4.94 & 14.67 \\
\hline & Chrysobalanaceae & 2.5 & 1.4 & 9.49 & 13.44 \\
\hline & Scytopetalaceae & 1.3 & 4.2 & 2.71 & 8.23 \\
\hline & Autres & 43.0 & 21.0 & 22.18 & 86.18 \\
\hline \multirow[t]{11}{*}{ Guiroutou } & Caesalpiniaceae & 10.6 & 19.3 & 13.22 & 43.15 \\
\hline & Apocynaceae & 4.3 & 14.2 & 9.10 & 27.54 \\
\hline & Euphorbiaceae & 11.7 & 8.6 & 4.24 & 24.59 \\
\hline & Mimosaceae & 4.3 & 7.3 & 4.99 & 16.56 \\
\hline & Ebenaceae & 3.2 & 7.8 & 5.24 & 16.19 \\
\hline & Olacaceae & 3.2 & 6.9 & 4.90 & 14.97 \\
\hline & Annonaceae & 6.4 & 4.2 & 2.83 & 13.42 \\
\hline & Scytopetalaceae & 1.1 & 2.4 & 8.23 & 11.74 \\
\hline & Rubiaceae & 5.3 & 3.3 & 2.26 & 10.90 \\
\hline & Octoknemaceae & 1.1 & 2.4 & 7.16 & 10.66 \\
\hline & Autres & 48.9 & 23.5 & 37.84 & 110.28 \\
\hline \multirow[t]{11}{*}{ Djapadji } & Ebenaceae & 6.1 & 22.2 & 7.48 & 35.74 \\
\hline & Euphorbiaceae & 12.2 & 10.5 & 10.85 & 33.57 \\
\hline & Mimosaceae & 4.9 & 7.5 & 13.33 & 25.69 \\
\hline & Caesalpiniaceae & 6.1 & 6.1 & 10.25 & 22.44 \\
\hline & Annonaceae & 6.1 & 8.9 & 4.10 & 19.07 \\
\hline & Olacaceae & 4.9 & 7.5 & 6.25 & 18.61 \\
\hline & Apocynaceae & 4.9 & 6.9 & 3.29 & 15.09 \\
\hline & Rubiaceae & 4.9 & 5.5 & 4.21 & 14.63 \\
\hline & Flacourtiaceae & 3.7 & 5.0 & 2.19 & 10.84 \\
\hline & Sterculiaceae & 2.4 & 1.9 & 4.60 & 8.98 \\
\hline & Autres & 43.9 & 18.0 & 33.44 & 95.35 \\
\hline \multirow[t]{8}{*}{ Sud PNT } & Caesalpiniaceae & 9.3 & 15.5 & 15.29 & 40.16 \\
\hline & Euphorbiaceae & 10.7 & 8.8 & 6.84 & 26.26 \\
\hline & Ebenaceae & 3.3 & 12.1 & 6.27 & 21.71 \\
\hline & Mimosaceae & 6.0 & 6.7 & 7.92 & 20.62 \\
\hline & Annonaceae & 6.7 & 7.7 & 5.03 & 19.42 \\
\hline & Olacaceae & 3.3 & 8.5 & 6.43 & 18.26 \\
\hline & Apocynaceae & 5.3 & 8.2 & 4.64 & 18.13 \\
\hline & Flacourtiaceae & 2.0 & 5.3 & 3.79 & 11.11 \\
\hline
\end{tabular}




\begin{tabular}{lcccc}
\hline Familles & $\begin{array}{c}\text { Diversité } \\
\text { relative }\end{array}$ & $\begin{array}{c}\text { Densité } \\
\text { relative }\end{array}$ & $\begin{array}{c}\text { Dominance } \\
\text { relative }\end{array}$ & VIF \\
\hline Sterculiaceae & 3.3 & 2.7 & 4.09 & 10.17 \\
Rubiaceae & 4.0 & 3.3 & 2.14 & 9.41 \\
Autres & 46.0 & 21.2 & 37.54 & 104.74 \\
\hline
\end{tabular}

Lorsqu'on compare les secteurs étudiés, Djapadji présente encore une particularité, contrairement aux deux autres secteurs. Il est le secteur dans lequel les Caesalpiniaceae ne sont pas parmi les 2 familles prépondérantes. Cette famille ne vient qu'en quatrième position des familles importantes. Les perturbations dans ce secteur ont certainement conduit à la réduction des représentants de cette famille.

\section{Discussion}

\section{4-1. Composition floristique et endémisme}

Le nombre insuffisant d'inventaires floristiques réalisés avant la présente étude dans le Parc National de Taï et plus particulièrement dans sa partie sud, limite les possibilités de comparaison. Nous pouvons cependant confronter nos résultats à ceux obtenus par Aké Assi et Pfeffer [18]. Ces auteurs ont recensé, lors d'un relevé itinérant dans l'ensemble du Parc, 868 espèces : 545 figurent dans notre liste et 323 ne s'y retrouvent pas. En revanche, dans le présent inventaire, 363 espèces qui n'avaient pas été recensées par ces auteurs ont été signalées. La richesse floristique du Parc National de Taii peut donc, dans l'état actuel des connaissances, être chiffrée à 1231 espèces. Nos résultats confirment toutes les projections sur la richesse spécifique du PNT et ses environs immédiats : environ 1300 espèces [12].

On peut affirmer que le PNT présente à lui seul le tiers de la richesse floristique nationale estimée à 3800 espèces [31]. Cependant les études et inventaires sont loin d'être complets. Notre inventaire a uniquement concerné trois secteurs dans le Sud. Certains habitats spéciaux, comme les collines amphibolitiques au centre de la zone d'inventaires ou les rives des cours d'eau, n'ont pas encore été prospectés. De la même manière, dans l'ensemble du PNT, toutes les zones Nord et Est restent quasiment inconnues pour la botanique. Enfin, il existe des travaux botaniques non encore répertoriés et des plantes provenant de Taii sont probablement présentes dans divers grands herbiers du monde à l'instar de ce que les travaux de [33] ont montré pour la forêt de Yapo-Abbé. La liste floristique exhaustive du PNT n'existe toujours pas.

On aurait pu s'attendre à ce que le secteur de Guiroutou, le plus arrosé, présente une plus grande richesse floristique que les deux autres secteurs. Notre technique de relevé 
ne l'a pas perçu. Cela est-il dô à la faible surface inventoriée? Les courbes aire-espèces tracées (Figure 2) montrent, en effet, une absence de palier pour tous les secteurs, ce qui, suggère que les aires minimales n'ont pas été atteintes.

L'endémisme est communément considéré comme un critère important pour évaluer la priorité et l'état de conservation d'un espace donné. Les espèces endémiques sont hautement vulnérables à la perturbation humaine et aux autres formes de changements environnementaux. La présence en grand nombre de ces plantes est généralement signe d'une grande biodiversité. Et leur distribution est fréquemment utilisée pour indiquer des 'Hotspots' (points chauds) de biodiversité $[34,35]$. Le nombre d'espèces endémiques ivoiriennes, ouest-africaines et de "sassandriennes", montre bien le niveau élevé de la diversité floristique dans le Parc en général et dans sa partie Sud, en particulier

L'une des explications de l'occurrence des espèces endémiques, dans le Parc et dans sa Partie Sud, est sans doute son appartenance au "Igroupe Côte d'Ivoirell l'un des 5 grands refuges forestiers d'Afrique [36]. Dans ce genre de site, selon Barbault et Sastrapradja [37] et Hawksworth et Kalin-Arroyo [38], la seule combinaison de l'histoire climatique et géologique, des facteurs écologiques contemporains et des propriétés biologiques inhérentes aux taxa et leurs combinaisons, ont dû contribuer à la spéciation et à la survie de ces espèces. De plus le Parc National de Taï fait partie du centre d'endémisme de la Haute Guinée.

Finalement, notre étude confirme bien que le PNT contient une assez forte proportion d'espèces exceptionnelles et que le Sud du Parc contribue grandement à cette richesse. Notre analyse confirme du même coup la thèse selon laquelle la zone d'étude comme la forêt classée de la Haute Dodo, constitue vraisemblablement un refuge pour quantité d'espèces qui avaient probablement une aire d'extension plus grande, pendant les périodes paléoclimatiques plus clémentes [7].

\section{4-2. Importances relatives des espèces et familles}

Le calcul des IVI a révélé 5 espèces à valeurs d'importance élevées et qui, de ce fait, sont prépondérantes dans la végétation du Sud-PNT. Ce sont des espèces qui se rencontrent en grande population dans la zone d'étude. Hymenostegia afzelii et Funtumia africana qui apparaissent pourtant dans un nombre restreint de placettes sont des espèces typiquement grégaires.

La dominance des Caesalpiniaceae et des Euphorbiaceae au niveau du VIF, nous amène à conclure que d'une façon générale la forêt du Sud du PNT, peut être caractérisée de 'type' à Caesalpiniaceae et Euphorbiaceae du fait des premières places que ces taxons occupent par leurs VIF respectifs. Parmi les six familles les plus Importantes du massif forestier de Yapo-Abbé identifiées par Corthay [33]: Burseraceae, Caesalpiniaceae, Euphorbiaceae, Meliaceae, Sapotaceae et Ebenaccae, seules les 
Caesalpiniaceae et les Euphorbiaceae ont des VIF élevés et occupent respectivement les $1^{1 \text { ère }}$ et $2^{\text {ème }}$ places dans la forêt du Sud du PNT Avec 49,41 comme VIF le plus élevé de notre étude, la forêt du Sud du PNT possède l'une des grandes valeurs du Tableau 6.

De nombreux travaux comme celui de Mori et Boom [39] montre qu'avec un VIF $>50$ une famille a toujours la valeur d'importance la plus élevée [33]. Les résultats de ce travail confirment cette affirmation. La famille des Caesalpiniaceae, avec un VIF de 49,41 , est la plus prépondérante famille, en ce qui concerne les grandes plantes $(\mathrm{dbh} \geq 10 \mathrm{~cm})$.

A l'exception de quelques différences, le sud-PNT présente les mêmes caractéristiques en général que la plupart des forêts africaines. Elle présente également les mêmes caractéristiques que la plupart des forêts néo-tropicales, en ce qui concerne les familles les plus importantes.

\section{Conclusion}

La flore du Sud du Parc National de Taï, telle que nous l'avons inventoriée, est riche de 908 espèces qui se répartissent entre 550 genres et 116 familles. Cette flore est essentiellement composée de phanérophytes avec une place non négligeable pour les lianes. Du point de vue du nombre d'espèces, cette forêt est floristiquement riche en Rubiaceae, Euphorbiaceae, Annonaceae et Apocynaceae lorsqu'on considère les plantes de toutes dimensions. Elle est également riche en espèces endémiques (175 espèces). Elle renferme 61 des 80 espèces "sassandriennes" qui se trouveraient dans le Parc selon [6].

De même, la forêt possède un important cortège d'espèces à statut particulier que sont les espèces rares, devenues rares, menacées d'extinction et vulnérables citées par [32] pour la flore de la Côte d'Ivoire. Ces espèces, au nombre de 11 représentent environ $1,2 \%$ de toutes les espèces recensées lors de cette étude. Le nombre d'espèces endémiques, "sassandriennes" et à statut particulier que contient cette flore, représenterait sa caractéristique particulière majeure par rapport aux autres forêts ivoiriennes. Cette particularité confirme bien que le PNT appartient aux Guinean Forests of West Africa Hotspot.

La diversité floristique des plantes de grands diamètres $(\mathrm{dbh} \geq 10 \mathrm{~cm})$ de la forêt du sud-PNT révèle que sur les 155 espèces de grandes tailles, les dominantes sont Hymenostegia afzelii, Strombosia pustulata, Diospyros sanza-minika, Funtumia africana, Calpocalyx brevibracteatus et Xylopia quintasii; ces espèces sont les plus fréquentes et tiennent une place importante dans cette flore.

Le VIF a permis de montrer que ce sont les familles des Caesalpiniaceae, Euphorbiaceae, Mimosaceae, Ebenaceae, Olacaceae, Annonaceae et Apocynaceae qui sont 
les plus diversifiées. La dominance des 4 premières familles fait du Sud de Taï un type de forêt à Caesalpiniaceae, Euphorbiaceae, Mimosaceae et Ebenaceae.

\section{Remerciements}

Nous voudrions remercier le Professeur Aké Assi pour avoir accepté de déterminer et vérifier la plupart des espèces. Notre gratitude va à l'endroit des guides et techniciens de terrain qui nous ont été d'une aide précieuse. Merci également aux personnes anonymes qui ont révisé cet article.

\section{Références}

[1] - G. Mangenot, "Etude sur la forêt des plaines et plateaux de Côte d'lvoire ॥. Etudes éburnéennes. I.F.A.N., Dakar, tome 4, (1956), pp 5-61

[2] - A. Aubréville, II A la recherche de la forêt en Côte d'Ivoire ॥. Bois et For. Trop. 56 (1958), pp. 12-32

[3] - G. A. Allport, C. Boesch, G. Couturier, J. Esser, G. Merz et J. Piart, I La faune [du PN Taii]. pp 72-93 in : E. P., Riezebos, A. P.Vooren, et J. L. Guillaumet (éds.): Le Parc National de Taï, Cöte d'lvoire. Synthèse des connaissances. La Fondation Tropenbos, Wageningen, Pays-Bas (1994)

[4] - S. D. Davis, V. H. Heywood, and A. C. Hamilton, "Centers of Plant Diversity: A Guide and Strategy for Their Conservation", (Volume 1). World Wilde Fund for Nature and IUCN-The World Conservation Union, Gland, Switzerland (1994)

[5] - C. Boesch et H. Boesch-Ackermann, "The Chimpanzees of the Taï Forest: Behavioural Ecology and Evolution". Oxford University Press, Oxford, UK, (2000)

[6] - J. L. Guillaumet, I Recherches sur la végétation et la flore de la région du BasCavally (Côte d'Ivoire) II. Mémoires ORSTOM no. 20, ORSTOM, Paris, (1967), p. 247

[7] - Ch. Huttel, I Etude de quelques caractéristiques structurales de la végétation du bassin versant de l'Audrénisrou II. Rapport ORSTOM, Adiopodoumé, (1977), p. 33

[8] - Van Rompaey, R.S.A.R. "Forest gradients in West Africa. A spatial gradient analysis". Doctoral thesis, Department of Forestry, Agricultural University Wageningen, Pays-Bas, (1993), p. 142

[9] - C. Boesch, "Hunting behaviour of wild chimpazees in Taï National Park". Am. J . Phys. Anthropol.78 (1989), pp 547-573

[10] - C. Boesch, "Social grouping in Taï chimpanzees". pp. 101-113 in: W.C. Mc Grew, L. F. Marhant and T. Nishida (ed.), Great Apesocieties. Cambridge: Cambridge Univ. Press, (1996) 
[11] - Z. B. Gone Bi, "Phénologie et distribution des plantes dont divers organes (principalement les tiges) sont consommés par les Chimpanzés, dans le Parc National de Taï II, Mém. DEA, Université de Cocody, (1999), p. 102

[12] - C. B. Châtelain, B. Kadjo, K. Inza et J. Refisch, II Relations Faune Flore dans le Parc National de Taï: une étude bibliographique II. Tropenbos - Côte d'Ivoire Séries 3, (2001), p. 166

[13] - E. P. Riezebos, (1994) ॥ Espace Taïll, in : " Le Parc National de Taï, Côte d'Ivoire. Synthèse de connaissances II. Tropenbos série 8. Wageningen, Pays-Bas, (2001) Eds. Riezebos, E.P., Vooren, A.P. et Guillaumet, J.-L.

[14] - M. Eldin, "Le climat "I. pp 77-108 in: Avénard, J.M., Eldin, M., Girard, G., Sircoulon, J., Touchebeuf, P., Guillaumet, J.L., Adjanohoun E., Perraud, A. Le milieu naturel de la Côte d'Ivoire. Mémoires ORSTOM, N50, Paris, France, (1971), p. 392

[15] - Van Rompaey, R.S.A.R. - Le climat. Pp $42-50$ in: Riezebos, E.P., Vooren, A.P. et Guillaumet, J.L. (éd.): Le Parc National de Taï, Côte d'Ivoire. Synthèse des connaissances. Tropenbos Serie 8. Waganingen, Pays-Bas, (1994)

[16] - Avénard, J. M. - Aspects de la géomorphologie. Pp 7-72 in : Avénard, J.M., Eldin, M., Girard, G., Sircoulon, J., Touchebeuf, P., Guillaumet, J.L., Adjanohoun E., Perraud, A. Le milieu naturel de la Côte d'Ivoire. Mémoires ORSTOM n 50 , Paris, France, (1971), p. 392

[17] - Collinet, J., B. Monteny, et B. Pouyaud, - Le milieu physique. in: Recherche et aménagement en milieu forestier tropical humide : le Projet Taï de Côte d'lvoire, (éd.) Guillaumet, J.L., Couturier, G. et Dosso, H. Notes du MAB, $n^{\circ}$ 15. U.N.E.S.C.O., Paris, France, (1984), p. 245

[18] - Aké Assi, L. et P. Pfeffer - Etude d'aménagement touristique du Parc National de Taï. Tome 2: Inventaire de la flore et de la faune. BDPA, Paris, (1975), p. 58

[19] - Hall, J.B. et M.D. Swaine - Distribution of vascular plants in tropica rain forest : Forest vegetation in Ghana. Dr.W. Junk Publishers, The Hague, Netherlands, (1981)

[20] - Hawthorne W. et M. Abu-Juam - Forest protection in Ghana. IUCN, Gland, (1995), p. 186

[21] - Hawthorne W.D. - Holes and the sums of parts in Ghanaian forest: regeneration, scale and sustainable use. Proceedings of the Royal Soc. Edinburgh 104B: (1996), pp. 75-176

[22] - Cheek, M. et Cable, S. - Plant inventory for conservation management: the Kew Earth-match Programme in Western Cameroon. (1996)

[23] - Aké Assi, L. - Flore de Côte d'Ivoire I, catalogue systématique, biogéographie et écologie. Conservatoire et Jardin Botaniques, Genève, Switzerland, (2001), p. 396

[24] - Aké Assi, L. - Flore de Côte d'lvoire 2, catalogue systématique, biogéographie et écologie. Conservatoire et Jardin Botaniques, Genève, Switzerland, (2002), p. 401 
[25] - Jongkind, C. C. H. - Checklist of Upper Guinea forest species. Pp 447-477 in: Poorter, L., Bongers, F., Kouamé, F. N'. et Hawthorne, W. D. (eds): Biodiversity of West African Forests: An ecological Atlas of Woody Plant Species. CABI Publiching, London, UK, (2004)

[26] - Curtis, J. T. et R. P. Macintosh -The interrelations of certain analytic and synthetic phytosociological characters. Ecology 31 (3), (1950), pp. 435-455

[27] - Mori, S.A., B.M. Boom, A.M. De Carvalino, et T.S. Dos Santos - Southern Bahia moist forest. Bot. Rev. 49(2) (1983) pp. 155-232

[28] - Kouamé, N. F. - Influence de l'exploitation forestière sur la végétation et la flore de la forêt classée du Haut-Sassandra (Centre-Ouest de la Côte d'Ivoire). Thèse de troisième cycle univ. de Cocody (Côte d'Ivoire), (1998), p. 227

[29] - Cottam, G. and J.T Curtis - The use of distance measures in phytosociological sampling. Ecology 37(1956), pp. 451-460

[30] - Bakayoko, A. - Comparaison de la composition floristique et de la structure forestière de parcelles de la forêt classée de Bossématié, dans l'Est de la Côte d'Ivoire. Mém. DEA, Univ. Cocody, Abidjan, (1999), pp. 72. + annexes

[31] - Aké Assi, L. - Flore de la Côte d'Ivoire: étude descriptive et biogéographique, avec quelques notes ethnobotaniques. Thèse Doct., Université d'Abidjan, Fac. des Sciences, Dépt. Phys. Végétale, (1984), p. 1206

[32] - Aké Assi, L. - Espèces rares et en voie d'extinction de la flore de la Côte d'Ivoire. Monogr. Syst. Bot. Missouri Bot. Gard. 25 (1988), pp. 461-463

[33] - Corthay R. - Analyse floristique de la forêt sempervirente de Yapo (Côte d'Ivoire). Mém. Diplôme, Univ. Génève, (1996), p. 152

[34] - Myers, N. - Threatened biotas 'hotspots' in tropical forests. The Environmentalist 8 (1988), pp. 187-208

[35] - Heywood, V.H. et R.T. Watson - Global biodiversity assessment. UNEP. Cambridge University Press, UK (1995)

[36] - Mayr, E. et R.J. O'Hara - The biogeographic evidence supporting the Pleistocene forest refuge hypothesis. Evolution 40. (1986), pp. 55-67

[37] - Barbault, R. et S. Sastrapradja - Generation, maintenance and loss of biodiversity. Pp 192-294 in: Heywood, V.H. et Waston, R.T. (eds) - Global biodiversity assessment. UNEP. Cambridge University Press, UK, (1995)

[38] - Hawksworth, D.L. et M.T. Kalin-Arroyo - Magnitude and distribution of biodiversity. In Heywood, V.H. \& Waston, R.T. (eds) - Global biodiversity assessment: 108-191. UNEP. Cambridge University Press, UK, (1995)

[39] - Mori, S.A. et B. M. Boom, - The Lecytrhidaceae of a lowland Neotropical forest. La fumée Mountain, French Guiana. Chapter II. The forest. Mem. New York Bot. Gard. 44, (1987), pp. 9-29 